> La signalisation calcique est essentielle pour les fonctions des lymphocytes T (LT), y compris celles des lymphocytes Th2 (T helper 2). Les lymphocytes Th2 produisent les interleukines 4, 5 et 13 et sont impliqués dans les maladies allergiques. L'activation des LT est à l'origine d'une entrée de $\mathrm{Ca}^{2+}$ via les canaux ORAl, et vraisemblablement d'autres canaux. Parmi eux, les canaux dépendant du voltage $\left(\mathrm{Ca}_{v} \mathrm{l}\right)$ sont décrits dans certains LT dont les Th2 où leur rôle a été démontré. Comme corollaire, l'inhibition des canaux $\mathrm{Ca}_{v} \mathrm{l}$ prévient l'asthme allergique expérimental impliquant les Th2. Dans cette revue, nous discuterons la singularité des réponses calciques selon le type de LT. <

\section{Les lymphocytes T}

Les lymphocytes T (LT) orchestrent la réponse immunitaire. Ils comprennent les LT helper (Th), qui expriment la molécule de surface CD4, et les LT cytotoxiques, qui expriment la molécule de surface CD8. Après leur rencontre avec la cellule présentatrice d'antigène chargée avec le peptide adéquat, les Th naïfs se différencient en cellules effectrices Th1, Th2 ou Th17 qui produisent des cytokines bien définies et exercent des fonctions différentes. Les Thl produisent de l'interféron- $\gamma$ et contribuent à l'élimination des virus tandis que les Th2 synthétisent de l'interleukine (IL)-4, de l'IL-5 et de I'IL-13 et contribuent à l'éradication des parasites. Les Th17 produisent de l'IL-17 et participent à la clairance des pathogènes. Les Th régulateurs (Teg), quant à eux, limitent la réponse immunitaire. Non seulement ces LT expriment des récepteurs pour des cytokines différentes qui induisent des voies de signalisation en aval qui sont donc différentes, mais aussi la signalisation en aval du récepteur T pour l'antigène (TCR) n'est pas identique dans les différents types de LT effecteurs.

Voir également dans ce numéro le débat entre Alain Trautmann (page 781) et L. Pelletier et M. Moreau (GDRE 731) (page 783).

Photo ci-dessus : lymphocytes Th2 exprimant des canaux Cav1.2 (encadré) pouvant causer un asthme expérimental marqué par des infiltrats pulmonaires d'éosinophiles (flèches) (๔ Lucette Pelletier).

\title{
La signalisation calcique dans les lymphocytes T
}

Virginie Robert ${ }^{1}$, Emily Triffaux $^{1}$, Magali Savignac ${ }^{1,2}$, Lucette Pelletier ${ }^{1,2}$

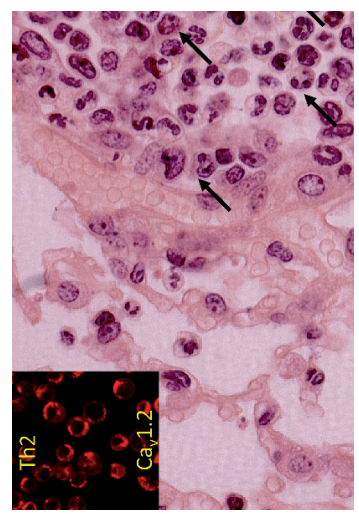

${ }^{1}$ Inserm U1043, centre de physiopathologie de Toulouse Purpan, place du Docteur Baylac, BP 3028, 31024 Toulouse Cedex 3 ; CNRS U5282, université de Toulouse Paul Sabatier, Toulouse 31300, France ; ${ }^{2}$ GDR 2688, calcium et régulation des gènes dans les conditions normales et pathologiques, France.

lucette.pelletier@inserm.fr virginie.robert@inserm.fr

\section{La signalisation calcique dans les LT}

Le $\mathrm{Ca}^{2+}$ est un second messager qui contrôle la plupart des processus biologiques. La concentration de $\mathrm{Ca}^{2+}$ dans le cytosol $\left([\mathrm{Ca}]_{i}\right)$ est étroitement régulée dans les LT, que ce soit en condition basale (environ $50 \mathrm{nM}$ ) ou après activation du TCR (jusqu'à $l \mu \mathrm{M}$ ). Le réticulum endoplasmique $(R E)$ contient la majorité des stocks de $\mathrm{Ca}^{2+}$ intracellulaire et la concentration calcique y est d'environ $400 \mu \mathrm{M}[1]$, tandis que les mitochondries tamponnent le $\mathrm{Ca}^{2+}$ cytosolique ${ }^{1}$. L'augmentation de la $[\mathrm{Ca}]_{i}$ peut être induite par la libération des stocks de $\mathrm{Ca}^{2+} \mathrm{du} R \varepsilon$ et/ou une entrée de $\mathrm{Ca}^{2+}$ extracellulaire via des canaux calciques. Certains canaux calciques exprimés à la membrane plasmique sont activés par la déplétion des stocks intracellulaires de $\mathrm{Ca}^{2+}$, tels que les canaux ORAI [31]. D'autres sont activés en réponse à la dépolarisation de la membrane (canaux $\left(a_{v}\right)$. L'activation du TCR induit le recrutement et l'activation de tyrosine kinases (TK) permettant la formation d'une plateforme de signalisation et l'activation de voies de signalisation en aval [2,3] (Figure 1). Parmi elles, la phospholipase C $\gamma$ (PLC $\gamma$ ) est activée, générant de l'inositol 1,4,5-triphosphate (IP3) et du diacylglycérol (DAG). L'IP3 se fixe à ses récepteurs (RIP3) induisant la libération des stocks de $\mathrm{Ca}^{2+} \mathrm{du} R E$ dans le cytosol. La diminution de concentration de $\mathrm{Ca}^{2+}$ dans le $\mathrm{RE}$ induit un changement conformationnel de STIMI (stromal interaction molecule) un senseur du $\mathrm{Ca}^{2+} \mathrm{du} \mathrm{RE}$-, son oligomérisation et sa migration au voisinage de la membrane plasmique. STIMl va alors activer les canaux ORAIl [31]. L'augmention de la $[\mathrm{Ca}]_{i}$ peut activer les calmoduline kinases et

\footnotetext{
${ }^{1}$ On désigne ainsi le fait que la $\left[\mathrm{Ca}^{2+}\right]$ peut s'élever au sein de la mitochondrie lors de la vidange du RE et que les mitochondries apposées au RE peuvent modifier le degré de l'influx de $\mathrm{Ca}^{2+}$ lors de la stimulation
} par un agoniste. 


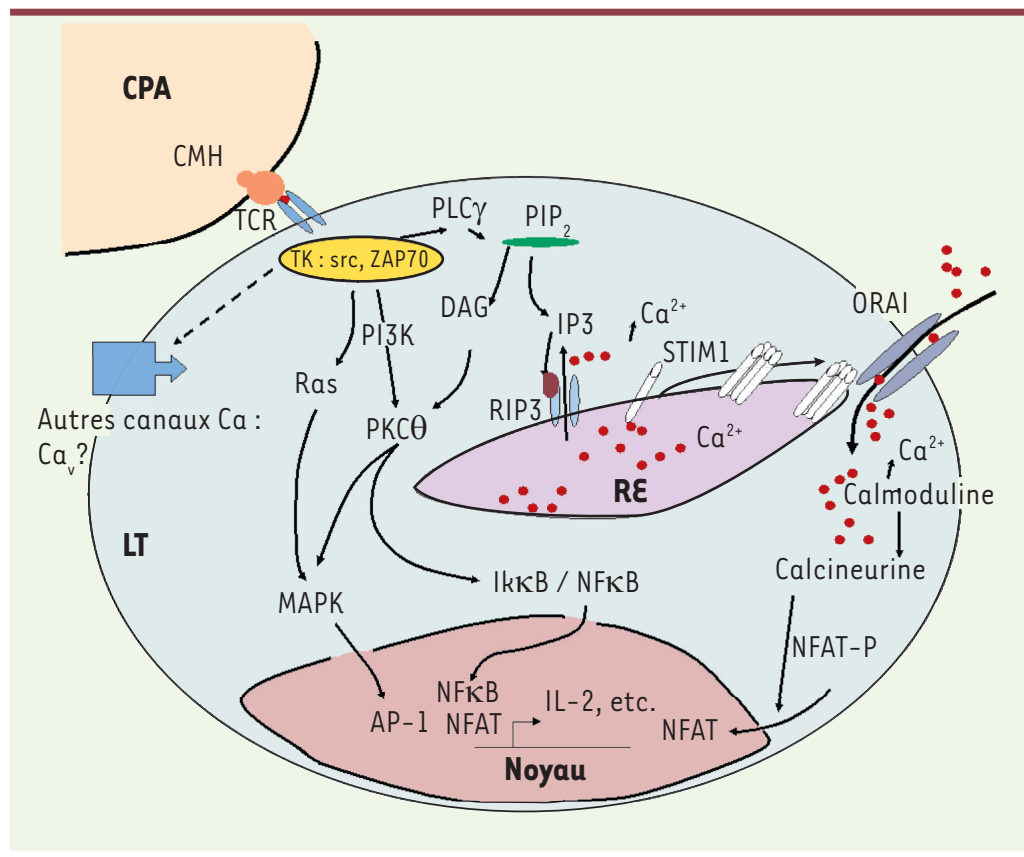

Figure 1. Signalisation calcique dans les lymphocytes $T$. La reconnaissance du peptide antigénique en association avec les molécules du complexe majeur d'histocompatibilité (CMH) à la surface de la cellule présentatrice d'antigène (CPA) par le récepteur T pour l'antigène (TCR) active les tyrosine kinases (TK) des familles src et syk (spleen tyrosine kinase) qui phosphorylent des enzymes incluant les MAP kinases, la phospholipase $C \gamma(P L C \gamma)$. Cette dernière clive le PIP2 en IP3 et diacylglycérol (DAG). L'IP3 se fixe sur ses récepteurs RIP3 à la membrane du $R E$ ce qui libère les stocks de $\mathrm{Ca}^{2+}$ du RE dans le cytosol. La déplétion est perçue par STIM qui s'oligomérise et vient en apposition avec les canaux ORAl ce qui permet un influx de $\mathrm{Ca}^{2+}$ du milieu extracellulaire dans le cytosol. D'autres canaux calciques comme les canaux $\mathrm{Ca}_{\mathrm{v}} \mathrm{l}$ pourraient contribuer à cet influx. L'augmentation de $[\mathrm{Ca}] \mathrm{i}$ active des calmoduline kinases et la calcineurine, une phosphatase qui déphosphoryle le facteur de transcription NFAT, permettant sa translocation nucléaire et l'activation des gènes cibles. PKC $\theta$ : protéine kinase $C \theta$; AP-1 : activator protein-1.

la calcineurine, une phosphatase dépendante du $\mathrm{Ca}^{2+}$ qui déphosphoryle les facteurs de transcription NFAT (nuclear factor of activated $T$ cells) permettant leur translocation nucléaire. Ces facteurs de transcription se fixent alors à leurs sites de liaison sur l'ADN au niveau des promoteurs des gènes cibles. Le plus souvent, NFAT agit en coopération avec d'autres facteurs de transcription pour déclencher la transcription de gènes définis selon la sous-population de LT. II est maintenant classiquement admis que l'activation de NFAT dépend d'une entrée de $\mathrm{Ca}^{2+}$ via les canaux ORAI dans les LT.

Toutefois, ce schéma de la régulation de la [Ca]i ne tient compte ni de l'existence d'autres canaux calciques, ni des différences de régulation de la $[\mathrm{Ca}]_{i}$ dans les populations de LT. Ainsi, la $[\mathrm{Ca}]_{i}$ est plus basse au repos dans les Thl que dans les Th2 mais après activation, l'augmentation de $[\mathrm{Ca}]_{i}$ est plus faible dans les Th2 que dans les Th1 [4]. Dans le même sens, Fanger et al. [5] ont montré que la clairance $\mathrm{du} \mathrm{Ca}^{2+}$ cytosolique est diminuée dans les Th2 par comparaison avec des Thl. Weber et al. [6] montrent que le pic initial de réponse calcique à la suite de l'engagement du TCR est le même dans les Thl, Th2 et les Th17, mais la décroissance est plus rapide dans les Th2 que dans les Thl avec des valeurs intermédiaires dans les Th17 (Figure 2). II serait intéressant d'explorer de façon plus systématique la réponse calcique dans les Th1, Th2 et Th17, en fonction de la façon dont ils ont été obtenus. En effet, les conséquences de ces différences de signaux calciques dans les populations de Then termes de singularité de réponses biologiques ne sont pas non plus connues. Toutefois, ces données montrent que la régulation de la $[\mathrm{Ca}]_{\mathrm{i}}$ dépend du type de LT, ce qui pourrait s'expliquer par des différences dans les voies de signalisation associées au TCR, et/ou à un équipement différentiel en canaux, pompes calciques ou autres canaux ioniques (cationiques non spécifiques, potassiques).

\section{Les canaux calciques dans les compartiments intracellulaires}

\section{Récepteurs IP3, $\mathrm{Ca}^{2+}$ blips et $\mathrm{Ca}^{2+}$ puffs}

Les RIP3 sont exprimés dans la plupart des cellules animales et sont un lien essentiel entre de nombreux récepteurs à la membrane plasmique qui stimulent la PLC et la libération de stocks intracellulaires de $\mathrm{Ca}^{2+}$ [7-9]. Chez les mammifères, les trois types de RIP3 sont structurellement proches et s'assemblent en homo ou hétérotétramères pour former des canaux calciques intracellulaires. Le rôle majeur du RIP3 est lié à sa localisation dans la membrane du $R E$, où il permet le passage de $\mathrm{Ca}^{2+}$ du RE dans le cytosol. Les RIP3 ont une sélectivité plutôt faible pour le $\mathrm{Ca}^{2+}$ par rapport à d'autres cations. Ce manque de sélectivité pourrait faciliter une libération rapide de $\mathrm{Ca}^{2+} \mathrm{du} R \varepsilon$ dans le cytosol en échange du passage de $\mathrm{K}^{+}$dans la direction opposée, ce qui maintiendrait le flux électrogénique de $\mathrm{Ca}^{2+}[10]$. Chaque RIP3 pourrait permettre le passage de 4500 à 15000 ions $\mathrm{Ca}^{2+}$ pendant son temps d'ouverture d'environ $10 \mathrm{~ms}$ [11]. Tous les RIP3 sont régulés à la fois par l'IP3 et le $\mathrm{Ca}^{2+}$. Ceci permet d'enclencher et propager des signaux calciques intracellulaires. Les libérations élémentaires de $\mathrm{Ca}^{2+}$ provoquées par l'IP3 dans les cellules sont le fait d'un très petit nombre de RIP3 actifs et de leurs interactions, qui dépendent $\mathrm{du} \mathrm{Ca}^{2+}$. Le $\mathrm{Ca}^{2+}$ qui diffuse d'un RIP3 actif favorise l'ouverture des récepteurs voisins. Ce phénomène est appelé CICR (calcium-induced calcium release) et permet au signal calcique dû à l'ouverture d'un seul RIP3 $\left(\mathrm{Ca}^{2+}\right.$ blip, que l'on pourrait traduire par un spot de 


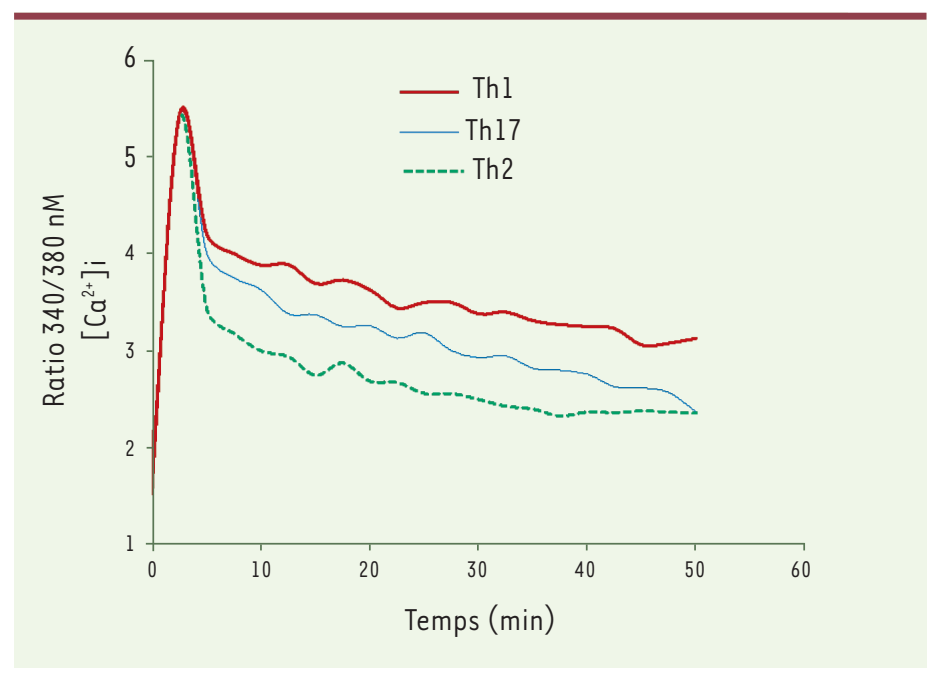

Figure 2. Analyse de la réponse calcique dans des lymphocytes Th1, Th2 et Th17. Des LT spécifiques d'un peptide de l'hémoglobine ont été différenciés en Thl, Th2 et Th17, chargés avec la sonde fluorescente sensible au $\mathrm{Ca}^{2+}$ Fura-2 AM [6]. Les cellules ont ensuite été stimulées avec des cellules présentatrices de l'antigène préincubées avec l'hémoglobine. Les cellules sont excitées aux longueurs d'onde 340 et $380 \mathrm{~nm}$, et l'émission est mesurée à la longueur d'onde $510 \mathrm{~nm}$. Le rapport de fluorescence obtenu en excitant à $340 \mathrm{nM}$ et $380 \mathrm{nM}$ est un indicateur de la concentration de $\mathrm{Ca}^{2+}$ intracellulaire. Les courbes présentées ne sont pas les courbes réelles, mais s'inspirent de celles publiées dans l'article de Weber et al. [6].

calcium) de croître en provoquant l'ouverture cordonnée de plusieurs RIP3 au sein d'un cluster. Cette majoration localisée de la libération de calcium, qui s'amplifie au fur et à mesure de l'augmentation de la concentration d'IP3, génère des «bouffées de calcium » $\left(\mathrm{Ca}^{2+}\right.$ puffs $)$, et peut même atteindre un seuil suffisant pour envahir la cellule entière $[8,12]$. Il est à noter que l'augmentation de la libération de $\mathrm{Ca}^{2+}$ générant les puffs n'est pas due à une augmentation directe de la quantité de calcium libérée par les RIP3, mais à l'augmentation de la fréquence d'ouverture de ces canaux [7].

\section{Signaux non calciques contrôlant les RIP3}

À côté du $\mathrm{Ca}^{2+}$ et de I'IP3, d'autres signaux intracellulaires peuvent réguler l'activité des RIP3 comme la fixation de nucléotides (ATP) et la phosphorylation par des kinases [13]. De façon intéressante, des kinases susceptibles de moduler l'activité des RIP3, comme la src kinase Fyn, sont activées par la stimulation du TCR et se localisent dans des complexes macromoléculaires contenant le RIP3 de type 1 (RIP3-1) qu'elles phosphorylent. L'aggrégation du RIP3-1 au site d'activation du TCR est due à un mouvement propre du canal vers la plateforme de signalisation associée au TCR [13]. La phosphorylation du RIP3-1 par Fyn augmente son affinité pour I'IP3 d'un facteur 5 et l'ouverture des canaux, même à des concentrations de $\mathrm{Ca}^{2+}$ normalement inhibitrices. Ainsi, les récepteurs RIP3-1 continueraient à relâcher du $\mathrm{Ca}^{2+}$ même quand la production d'IP3 décline, permettant une entrée continue de $\mathrm{Ca}^{2+}$ via les canaux ORAl et l'activation de NFAT [13].

\section{STIM et ORAI : le couplage entre le RE et la membrane plasmique}

STIMI est une protéine liant le calcium qui couple la déplétion des stocks calciques du RE à un influx de $\mathrm{Ca}^{2+}$ à partir du milieu extracellulaire via les canaux ORAI [14-16]. Ces canaux ont une sélectivité élevée pour le $\mathrm{Ca}^{2+}$ mais une conductance unitaire faible (passage d'environ $10^{4}$ ions $\mathrm{Ca}^{2+}$ par canal et par seconde à un potentiel de membrane de $100 \mathrm{mV}$ ) [17]. La déplétion des stocks de $\mathrm{Ca}^{2+}$ du RE libère le $\mathrm{Ca}^{2+}$ de STIM. II s'ensuit l'oligomérisation de STIMl et sa migration vers des zones proches de la membrane plasmique où il interagit avec ORAI, permettant l'ouverture du canal (Figure l). STIM et ORAI sont surtout localisés à proximité de la synapse immunologique, une zone de contact entre le LT et la cellule présentatrice de l'antigène qui est enrichie en molécules de signalisation [18]. L'importance d'ORAI est confirmée par l'observation d'une immunodéficience sévère chez l'homme lorsqu'existent des mutations spontanées dans le gène codant pour ORAll [15, 31]. Les souris invalidées pour les gènes codant ORAI ou STIM donnent des résultats déconcertants [17]. Ainsi, les souris invalidées à la fois pour STIM1 et STIM2 présentent une maladie auto-immune et inflammatoire sévère suggérant que l'absence de ces molécules a un impact profond sur les LT régulateurs qui contrôlent la réponse immunitaire, et moindre sur les autres populations de LT.

\section{Les canaux $\mathrm{Ca}_{v} 1$ : des canaux à part entière dans les LT ?}

\section{Structure et régulation des canaux $\mathrm{Ca}_{\mathrm{v}} \mathrm{l}$}

Les canaux $\mathrm{Ca}_{v} 1$ sont caractéristiques des cellules excitables - neurones, cellules cardiaques - où ils sont activés par de fortes dépolarisations de la membrane [19]. Ils sont formés par une sous-unité $\alpha l$ qui constitue le pore calcique et donne au canal ses caractéristiques biophysiques. La séquence en acides aminés est organisée en quatre domaines répétés (de I à IV), chacun contenant six segments transmembranaires (de S1 à S6) et une boucle extracellulaire entre les segments transmembranaires S5 et $\mathbf{S} 6$ de chaque domaine. Les segments $\mathbf{S} 4$ de chaque domaine servent de senseurs du voltage, et induisent un changement de conformation en cas de dépolarisation, ce qui ouvre le pore. Le segment S6 comporte les sites de fixation aux molécules antagonistes des canaux $\mathrm{Ca}_{\mathrm{v}} \mathrm{l}$ comme les dihydropyridines (DHP) couramment utilisées dans le traitement de l'hypertension artérielle. La sous-unité $\alpha$ l transmembranaire 


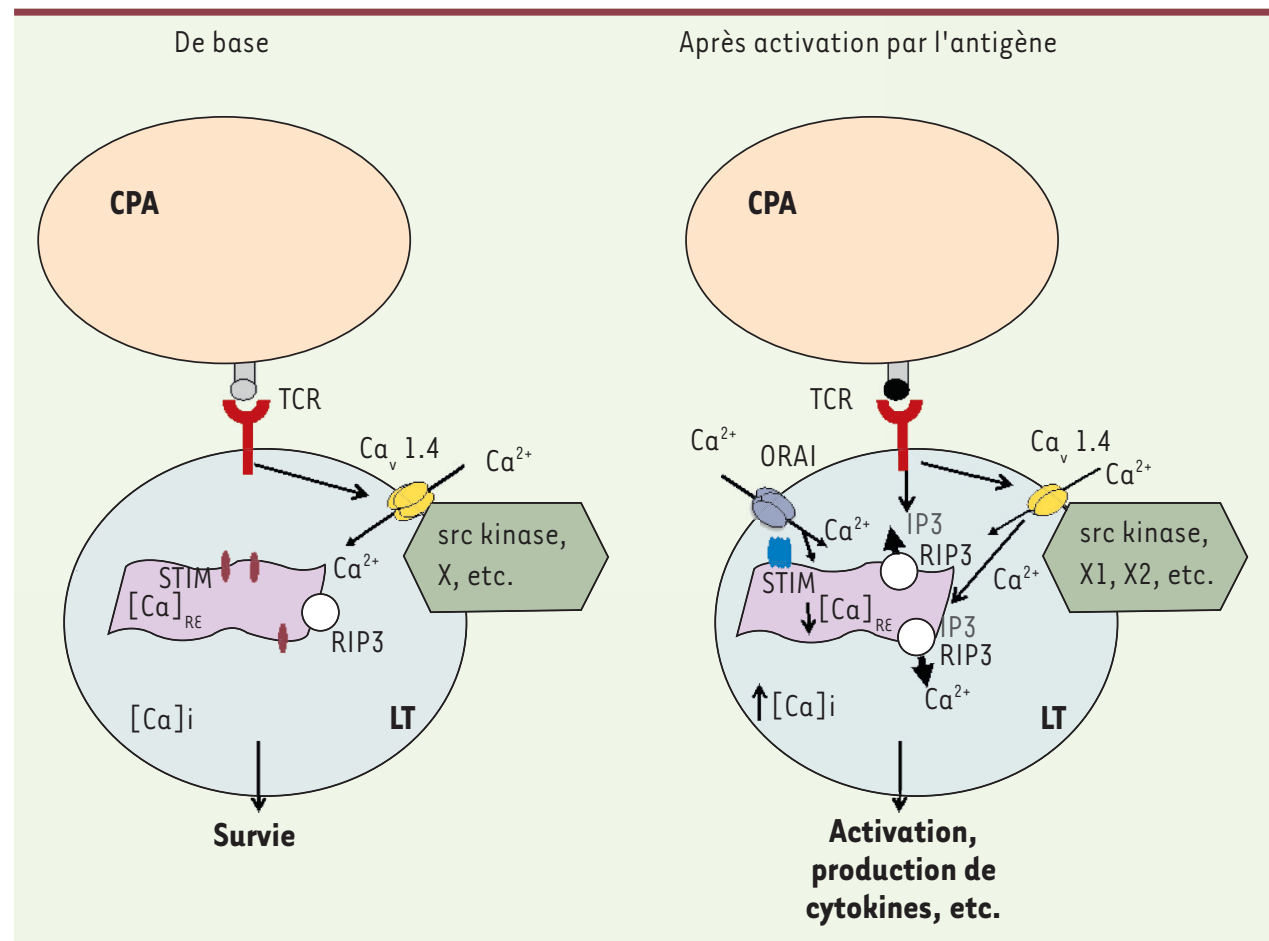

Figure 3. Rôle des canaux Cav1.4 dans les lymphocytes T. D'après les travaux des groupes de Flavell et Jefferies, les canaux $\mathrm{Ca}_{\mathrm{v}} \mathrm{l} .4$ seraient nécessaires à la survie des $\mathrm{LT}$ et leur activation. De base, la stimulation du TCR a minima, probablement due à l'interaction de molécules du complexe majeur d'histocompatibilité (CMH) associées avec des peptides du soi, serait responsable d'une entrée de calcium par des canaux $\mathrm{Ca}_{v} 1.4$ suffisante pour permettre la survie des LT. Ces canaux $\mathrm{Ca}_{v} 1.4$ se trouveraient au sein de complexes de signalisation déjà existants dans les LT avant toute activation. Ces complexes contiendraient une kinase src et d'autres protéines $(X)$ qui ne sont pas toutes encore identifiées. Lors de la reconnaissance du peptide antigénique spécifique, la stimulation du TCR entraînerait la production d'IP3 qui, en se fixant sur les RIP3 à la membrane du réticulum endoplasmique (RE), causerait une sortie du $\mathrm{Ca}^{2+} \mathrm{du} R \varepsilon$ vers le cytosol. Cette baisse de concentration de $\mathrm{Ca}^{2+}$ dans le RE $\left([\mathrm{Ca}]_{\mathrm{RE}}\right)$ serait perçue par STIM avec pour conséquence une entrée de $\mathrm{Ca}^{2+}$ du milieu extracellulaire dans le cytosol. Les canaux $\mathrm{Ca}_{v} \mathrm{l} .4$ contribueraient en partie à la réponse calcique en assurant une $[\mathrm{Ca}]_{R \varepsilon}$ correcte. Néanmoins, leur rôle exact reste à définir. La conséquence de l'activation permettra au LT de produire des cytokines et d'exercer ses fonctions.

est associée avec les sous-unités $\alpha 2 \delta$, $\beta$ et possiblement $\gamma$. Quatre gènes codent pour les sous-unités $\alpha$ l des canaux (de $\mathrm{Ca}_{\mathrm{v}} \mathrm{l} .1$ à $\left.\mathrm{Ca}_{\mathrm{v}} \mathrm{l} .4\right)$ dont l'expression est relativement spécifique de tissus. La diversité des canaux calciques est encore augmentée par le fait que quatre gènes codent pour les sous-unités $\beta$ [19] qui modulent les propriétés du canal. Les canaux $\left[a_{v}\right]$ se répartiraient entre plusieurs états qualifiés de mode 0 (inactivables même après de fortes dépolarisations de la membrane dans des gammes physiologiques), mode 1 (probabilité faible d'ouverture et temps d'ouverture bref après dépolarisation de la membrane) et mode 2 (probabilité d'ouverture plus élevée et temps d'ouverture plus long). Par exemple, la protéine kinase A activée par la stimulation $\beta$-adrénergique augmenterait le courant calcique passant par les canaux $\mathrm{Ca}_{\mathrm{v}} \mathrm{l} .2$ en modifiant la répartition des canaux en faveur des modes 1 et 2 [19].
Arguments en faveur d'un rôle des canaux $\mathrm{Ca}_{v} 1$ dans la fonction des lymphocytes $T$ et questions en suspens Plusieurs groupes ont montré que des inhibiteurs pharmacologiques comme les DHP, considérés comme spécifiques des canaux $\mathrm{Ca}_{v} \mathrm{l}$, diminuaient les fonctions biologiques de LT naïfs Th ou cytotoxiques (définis par l'expression du marqueur de surface $\left.\operatorname{CD} 8^{+}\right)$in vitro $[20$ 22]. Mais les drogues comme les DHP utilisées à des doses relativement élevées (> $1 \mu \mathrm{M}$ ) pourraient agir sur des canaux potassiques, ce qui expliquerait cet effet [23]. Des transcrits des sous-unités $\alpha$ l des canaux $\mathrm{Ca}_{v} \mathrm{l}$ sont détectés dans les $\mathrm{LT} \mathrm{CD}^{+}{ }^{+}$et CD8 $8^{+}$humains et de souris. Parfois, certains auteurs ont rapporté des formes tronquées expliquant, de ce fait, l'absence de sensibilité au potentiel des canaux dans les LT [21, 22]. Cependant, ces auteurs n'ont pas démontré le rôle fonctionnel de ces canaux tronqués, et la relation structure fonction reste à déterminer [21, 22]. Ces dernières années, l'étude de la réponse immunitaire de souris mutées spontanément dans le gène codant pour la sous-unité $\beta 4$ [24], de souris $\beta 3$ knockout [25] et, plus récemment, de souris invalidées pour la sous-unité $\alpha 1$ de $\mathrm{Ca}_{v} \mathrm{l} .4$ [26] plaident pour un rôle des canaux $\left.\mathrm{Ca}_{v}\right]$ dans les $\mathrm{LT} \mathrm{CD}^{+}{ }^{+}$et $\mathrm{CD} 8^{+}$, même si leur mode de fonctionnement n'est pas compris. Le travail le plus abouti, celui de Flavell et al. [25], montre que la perte d'expression de la sous-unité $\beta 3$ cause la perte d'expression de $\mathrm{Ca}_{v} 1.4$, prédominant dans les LT CD $8^{+}$qui n'ont jamais été activés. Ceci est associé à un défaut de survie des cellules (Figure 3) probablement en raison d'une trop forte expression de la molécule proapoptotique Fas. L'augmentation de $[\mathrm{Ca}]_{i}$ qui suit l'activation du TCR est réduite dans les $\mathrm{LT} C D 8^{+} \beta 3^{-1}$. Le fait que la réponse calcique en réponse à l'ionomycine soit inchangée dans 
A
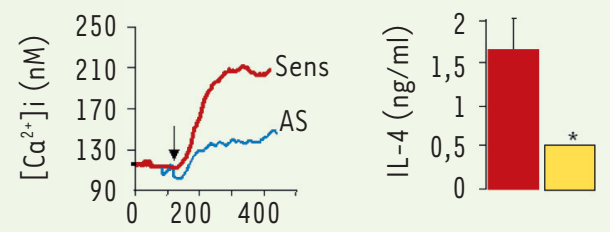

\section{C}

B

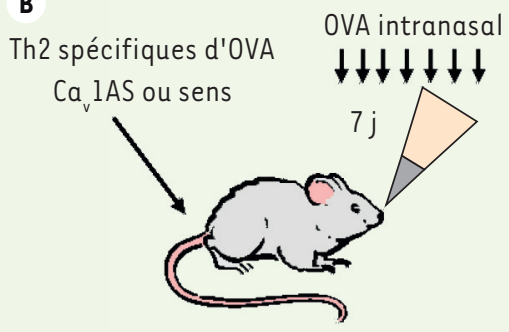

25
20
15
10
5
0

Total

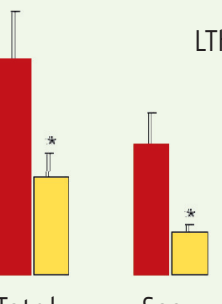

Eos
LTh2 transfectés avec:

$\mathrm{Ca}_{\mathrm{v}} \mathrm{lAS}$

$\mathrm{Ca}_{\mathrm{v}} \mathrm{l}$ sens

Figure 4. La perte d'expression des canaux Cav1 dans les Th2 abolit leurs fonctions et leur capacité à induire un asthme expérimental. A. Des lymphocytes T transgéniques pour un récepteur T (TCR) spécifique d'un peptide de l'ovalbumine (OVA) (souris D011.10) ont été différenciés en Th2 et transfectés avec des oligonucléotides (ON) anti-sens (AS) spécifiques des canaux Cavl ( $\mathrm{Ca}_{\mathrm{v}} \mathrm{IAS}$ ) ou des ON contrôles (sens). La transfection avec les $\mathrm{Ca}_{v} 1 \mathrm{AS}$ réduit de 50 à $70 \%$ l'expression des canaux $\mathrm{Ca}_{v} 1$ (non montré). Ces LT ont été stimulés par un anticorps anti-TCR et nous avons analysé $\mathrm{la}[\mathrm{Ca}]_{i}$ et la production d'interleukine (IL)-4, spécifique des Th2. La transfection avec $\mathrm{Ca}_{v}$ lAS réduit à la fois l'augmentation de [Ca $]_{i}$ et la production d'IL-4 après stimulation du TCR. B. Nous avons injecté ces Th2 transfectés avec $\mathrm{Ca}_{v}$ IAS ou les ON contrôles (sens) chez des souris BALB/c à qui nous avons fait respirer de l'OVA. C. Nous avons collecté les liquides de lavage bronchoalvéolaire pour analyser l'inflammation pulmonaire caractéristique de l'asthme allergique. Le nombre total de cellules inflammatoires, d'éosinophiles (Eos) et de monocytes (Mono) est significativement $(*)$ réduit chez les souris injectées avec les Th2 transfectés avec $\mathrm{Ca}_{v}$ lAS par comparaison avec les souris injectées avec les Th2 contrôles ( $\mathrm{Ca}_{v} \mathrm{l}$ sens). Lymphos : lymphocytes; Neutros : polynucléaires neutrophiles.

les $L T \beta 3^{-/-}$suggère que les canaux $C a_{v} 1$ ne sont pas impliqués dans l'entrée capacitative de calcium (associée à la déplétion des stocks intracytoplasmiques de $\mathrm{Ca}^{2+}$ ). La translocation nucléaire de NFAT est diminuée et surtout l'expression de la protéine NFATcl est très réduite dans les $L T C D 8^{+}$naïfs qui n'expriment pas la sous-unité $\beta 3$. Les auteurs démontrent que $C a_{v} 1.4$ est associé à la sous-unité $\beta 3$ avec des partenaires de signalisation comme Lck (lymphocyte-specific protein tyrosine kinase) et Vav dans des complexes préformés avant même l'engagement du TCR. Selon ces auteurs, l'interaction entre le TCR et les molécules du $\mathrm{CMH}$ qui présentent des peptides du soi induit une signalisation calcique basale dépendant de $\mathrm{Ca}_{v} 1.4$ et responsable de la survie (Figure 3). Ce qui se passe lors de l'activation et du passage d'expression de $\mathrm{Ca}_{v} 1.4$ à $\mathrm{Ca}_{v} 1.1$, la forme présente dans les LT activés [27], n'est pas explicité. Omilusik et al. [26] vont dans le même sens que Flavell et al. [25] mais explicitent des différences en ce qui concerne l'interaction entre entrée capacitative et canaux $\mathrm{Ca}_{v} 1.4$. Omilusik et al. [26] montrent un défaut de survie des LT CD $8^{+}$chez des souris invalidées pour $\mathrm{Ca}_{v} 1.4$. Ces auteurs détectent des courants calciques après dépolarisation de la membrane dans des LT préstimulés par le TCR, attestant la présence de canaux $\mathrm{Ca}_{v} \mathrm{l}$ à la membrane dans des LT activés. Ces courants sont absents dans les LT de souris $C a_{v} 1.4^{-1-}$. $\mathrm{Ca}_{V} 1.4$ semble requis pour l'augmentation de la $[\mathrm{Ca}]_{\text {i }}$ après stimulation par le TCR ou la vidange des stocks calciques intracellulaires, particulièrement dans les $\mathrm{LT} C D 4^{+}$naïfs et quasiment pas dans les LT CD4 $4^{+}$mémoires. L'ouverture des canaux $\mathrm{Ca}_{V} 1.4$ dépendrait dans les LT de la signalisation via le TCR et servirait à remplir les stocks intracellulaires de $\mathrm{Ca}^{2+}$ dans les LT naïfs et en cours de différenciation. La réponse calcique est aussi très diminuée dans les LT $\mathrm{CD}^{+}$mémoires de souris $\mathrm{Ca}_{v} 1.4^{-/-}$stimulés via le TCR, posant le problème de la part respective des canaux Cav 1.4 et d'ORAI.

\section{Les canaux $\mathrm{Ca}_{\mathrm{v}} 1$ : marqueurs de LT effecteurs}

Notre groupe a montré que les canaux $\mathrm{Ca}_{v} 1.2$ et $\mathrm{Ca}_{v} \mathrm{l} .3$ sont sélectivement induits dans les Th2 différenciés (qui sécrètent de l'IL-4), et disparaissent dans les Thl producteurs d'IFN- $\gamma[28,29]$. Les ARNm et les protéines de ces canaux sont détectables dans les Th2. La transfection des Th2 avec des oligodéoxynucléotides anti-sens $\left(\mathrm{Ca}_{v} \mathrm{lAS}\right)$ spécifiques des canaux $\mathrm{Ca}_{\mathrm{v}} 1.2$ et/ ou $\mathrm{Ca}_{v} 1.3$, entraîne la perte d'expression de ces sousunités et une forte diminution de l'augmentation de $\left[\mathrm{Ca}^{2+}\right]_{\mathrm{i}}$ et de la production de cytokines par les Th2 suite à l'engagement du TCR (Figure 4), tout en n'ayant aucun 
effet sur les Th1. Les Th2 transfectés avec les $\mathrm{Ca}_{v}$ lAS perdent leur capacité à induire un asthme dans des expériences de transfert adoptif [29] (Figure 4). Des Thl spécifiques de l'ovalbumine, transfectés ou non avec les $\mathrm{Ca}_{v} 1 \mathrm{AS}$, induisent la même inflammation pulmonaire quand ils sont transférés chez des souris exposées à l'ovalbumine par voie aérienne [29]. Enfin, l'inhalation de $\mathrm{Ca}_{v} \mathrm{lAS}$ prévient un asthme expérimental, indiquant que ces canaux pourraient représenter une nouvelle cible intéressante dans les maladies allergiques. L'absence de sensibilité au voltage des canaux $\mathrm{Ca}_{v} 1$ dans les Th2 pourrait s'expliquer par une régulation différente de celle classiquement établie dans les cellules excitables.

\section{Connexions entre canaux ORAl et canaux $\mathrm{Ca}_{\mathrm{v}} 1$ dans les LT ?}

Des travaux récents montrent une régulation coordonnée des canaux $\mathrm{Ca}_{v} 1$ et ORAI via STIMI [30]. La déplétion des stocks intracellulaires de $\mathrm{Ca}^{2+}$ qui induit la localisation de STIMl juste sous la membrane plasmique permettrait l'interaction du même domaine de STIM avec ORAI et $\mathrm{Ca}_{v}$ 1.2. En conséquence, l'activation d'ORAI par STIMI diminuerait l'influx de $\mathrm{Ca}^{2+}$ par les canaux $\mathrm{Ca}_{v} 1.2$. Ceci aurait des conséquences biologiques puisque l'influx de $\mathrm{Ca}^{2+}$ via les canaux $\mathrm{Ca}_{v} 1.2$ et ORAI n'active pas les mêmes voies.

II sera important dans le futur de comprendre la dynamique d'ouverture des canaux $\mathrm{Ca}_{v} \mathrm{l}$ et ORAl dans les cellules excitables et non excitables, y compris dans les LT. II faudra comprendre pourquoi certains types de LT expriment des canaux $\mathrm{Ca}_{v} 1$, et l'impact possible sur le programme génétique associé à leurs fonctions particulières. Il est possible que les flux de $\mathrm{Ca}^{2+}$ via les canaux $\mathrm{Ca}_{v} 1$ induisent une signature calcique particulière, essentielle pour le remodelage de la chromatine au niveau des gènes codant pour les cytokines produites par les Th2 par exemple (un processus régulé par le $\mathrm{Ca}^{2+}$ ). II est concevable de pouvoir inhiber un maillon contrôlant sélectivement la signalisation calcique dans une population de LT responsable d'un certain type de pathologie, ce qui permettrait de traiter des désordres immunologiques sans induire d'immunosuppression globale. $\diamond$

\section{SUMMARY}

\section{Calcium signaling in T lymphocytes}

Calcium signaling is essential for all the functions of T lymphocytes, including those of Th2 cells. Th2 lymphocytes producing interleukins 4,5 and 13 orchestrate allergic diseases including asthma. T-cell activation induces an influx of $\mathrm{Ca}^{2+}$ from the external medium through ORAI calcium channels although other calcium channels are likely to be involved. Among them, voltage-gated calcium $\left(\mathrm{Ca}_{v} \mathrm{l}\right)$ channels have been reported in some T-cell subsets including Th2 cells. The inhibition of $\mathrm{Ca}_{\mathrm{v}} \mathrm{I}$ channels abrogates $\mathrm{T}$-cell receptor-driven calcium influx and interleukin production by Th2 cells. From a therapeutic point of view, the inhibition of $\mathrm{Ca}_{v} 1$ channels prevents Th2-dependent experimental allergic asthma. In this review, we will discuss the singularities of calcium responses depending upon the T-cell subset and its state of activation. $\diamond$
LIENS D'INTÉRÊT

Les auteurs déclarent n'avoir aucun lien d'intérêt concernant les données publiées dans cet article.

\section{REMERCIEMENTS}

Ce travail a pu être réalisé grâce au financemement donné par l'association 111 des Arts et par la Société française d'allergologie (SFA). Virginie Robert est financée par une allocation du Ministère (MENRT) et Emily Triffaux par un projet de « Recherche clinique translationnelle » (Inserm-DGOS).

\section{RÉFÉRENCES}

1. Brandman 0, Liou J, Park WS, Meyer T. STIM2 is a feedback regulator that stabilizes basal cytosolic and endoplasmic reticulum $\mathrm{Ca}^{2+}$ levels. Cell 2007 ; $131: 1327-39$.

2. Smith-Garvin JE, Koretzky GA, Jordan MS. T cell activation. Annu Rev Immunol 2009; $27:$ 591-619.

3. Yokosuka T, Saito T. The immunological synapse, TCR microclusters, and T cell activation. Curr Top Microbiol Immunol 2010 ; 340 : 81-107.

4. Sloan-Lancaster J, Steinberg TH, Allen PM. Selective loss of the calcium ion signaling pathway in T cells maturing toward a T helper 2 phenotype. J Immunol 1997 ; 159 : 1160-8.

5. Fanger CM, Neben AL, Cahalan MD. Differential $\mathrm{Ca}^{2+}$ influx, $\mathrm{K}_{\mathrm{Ca}}$ channel activity, and $\mathrm{Ca}^{2+}$ clearance distinguish Thl and Th2 lymphocytes. J Immunol $2000 ; 164: 1153-60$.

6. Weber K, Miller MJ, Allen PM. Th17 cells exhibit a distinct calcium profile from Th1 and Th2 cells and have Th1-like motility and NF-AT nuclear localization. J Immunol 2008 ; 180 : 1442-50.

7. Taylor CW, Prole DL, Rahman T. $\mathrm{Ca}^{2+}$ channels on the move. Biochemistry $2009 ; 48: 12062-80$.

8. Taufiq Ur R, Skupin A, Falcke M, Taylor CW. Clustering of InsP3 receptors by InsP3 retunes their regulation by InsP3 and $\mathrm{Ca}^{2+}$. Nature $2009 ; 458: 655-9$.

9. Rossi A, Tovey S, Rahman T, et al. Analysis of IP(3) receptors in and out of cells. Biochim Biophys Acta 2011 ; 14 octobre (online).

10. Gillespie D, Fill M. Intracellular calcium release channels mediate their own countercurrent: the ryanodine receptor case study. Biophys J 2008 ; 95 : 3706-14.

11. Bezprozvanny I, Ehrlich BE. Inositol (1,4,5)-trisphosphate (InsP3)gated Ca channels from cerebellum: conduction properties for divalent cations and regulation by intraluminal calcium. J Gen Physiol 194 ; $104: 821-56$.

12. Smith IF, Wiltgen SM, Shuai J, Parker I. $\left.\mathrm{Ca}^{(2+}\right)$ puffs originate from preestablished stable clusters of inositol trisphosphate receptors. Sci Signal 2009 ; 2 : ra77.

13. Vanderheyden V, Devogelaere B, Missiaen L, et al. Regulation of inositol 1,4,5-trisphosphate-induced $\mathrm{Ca}^{2+}$ release by reversible phosphorylation and dephosphorylation. Biochim Biophys Acta 2009 ; 1793 : 959-70.

14. Roos J, DiGregorio PJ, Yeromin AV, et al. STIMl, an essential and conserved component of store-operated $\mathrm{Ca}^{2+}$ channel function. J Cell Biol $2005 ; 169$ : 435-45.

15. Feske $S$, Gwack Y, Prakriya M, et al. A mutation in Orail causes immune deficiency by abrogating CRAC channel function. Nature $2006 ; 441: 179-85$.

16. Vig $M$, Peinelt $C$, Beck et al. CRACMl is a plasma membrane protein essential for store-operated $\mathrm{Ca}^{2+}$ entry. Science 2006 ; 312 : 1220-3.

17. Hogan PG, Lewis RS, Rao A. Molecular basis of calcium signaling in lymphocytes: STIM and ORAI. Annu Rev Immunol 2010 ; 28 : 491-533.

18. Lioudyno MI, Kozak JA, Penna A, et al. Orail and STIMl move to the immunological synapse and are up-regulated during T cell activation. Proc Natl Acad Sci USA 2008 ; 105 : 2011-6.

19. Catterall WA. Structure and regulation of voltage-gated $\mathrm{Ca}^{2+}$ channels. Ann Rev Cell Dev Biol $2000 ; 16: 521-55$.

20. Stokes L, Gordon J, Grafton G. Non-voltage-gated L-type $\mathrm{Ca}^{2+}$ channels in human T cells: pharmacology and molecular characterization of the major alpha pore-forming and auxiliary beta-subunits. J Biol Chem 2004 ; 279 : 19566-73.

21. Kotturi MF, Jefferies WA. Molecular characterization of L-type calcium channel splice variants expressed in human T lymphocytes. Mol Immunol $2005 ; 42: 1461-74$. 


\section{RÉFÉRENCES}

22. Kotturi MF, Hunt SV, Jefferies WA. Roles of CRAC and $\mathrm{Ca}(\mathrm{V})$-like channels in T cells: more than one gatekeeper? Trends Pharmacol Sci $2006 ; 27: 360-7$.

23. Randriamampita C, Bismuth G, Debre P, Trautmann A. Nitrendipine-induced inhibition of calcium influx in a human T-cell clone: role of cell depolarization. Cell Calcium 1991; $12: 313-23$.

24. Badou A, Jha MK, Matza D, et al. Critical role for the beta regulatory subunits of Cav channels in T lymphocyte function. Proc Natl Acad Sci USA 2006 ; 103 : 15529-34.

25. Jha MK, Badou A, Meissner M, et al. Defective survival of naive CD8 ${ }^{+}$T lymphocytes in the absence of the beta3 regulatory subunit of voltage-gated calcium channels. Nat Immunol 2009; 10 : 1275-82.

26. Omilusik K, Priatel JJ, Chen $\mathrm{X}$, et al. The $\mathrm{Ca}\left({ }_{\mathrm{v}}\right) 1.4$ calcium channel is a critical regulator of $\mathrm{T}$ cell receptor signaling and naive T cell homeostasis. Immunity $2011 ; 35: 349-60$.

27. Matza D, Badou A, Kobayashi KS, et al. A scaffold protein, AHNAK1, is required for calcium signaling during T cell activation. Immunity $2008 ; 28: 64-74$

28. Djata Cabral M, Gomes B, Savignac M, et al. Signalisation dans les lymphocytes $T$ : implication de canaux calciques. Med Sci (Paris) $2007 ; 23: 136-8$.
29. Djata Cabral M, Paulet PE, Robert V, et al. Knocking-down Cavl calcium channels implicated in Th2-cell activation prevents experimental asthma. Am J Respir Crit Care Med 2010 ; 181 : 1310-7.

30. Park CY, Shcheglovitov A, Dolmetsch R. The CRAC channel activator STIMI binds and inhibits L-type voltage-gated calcium channels. Science 2010 $330: 101-5$.

31. Le Deist F, Capiod T. Immunodéficiences et pathologies associées aux mutations dans STIM/ORAI : un complexe membranaire au cœur de la signalisation calcique. Med Sci (Paris) $2011 ; 27$ : 737-45.
TIRÉS À PART

L. Pelletier

\section{Collection SCIENCE ET BIOMÉDECINE}

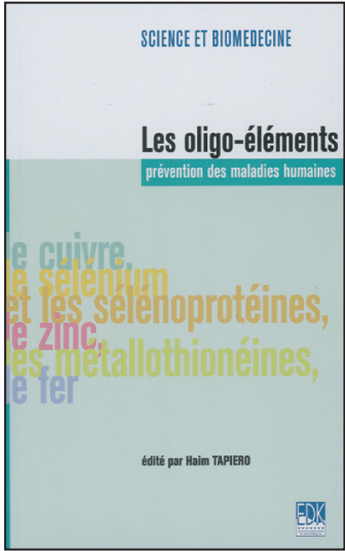

ISBN : 2-84254-107-3 64 pages

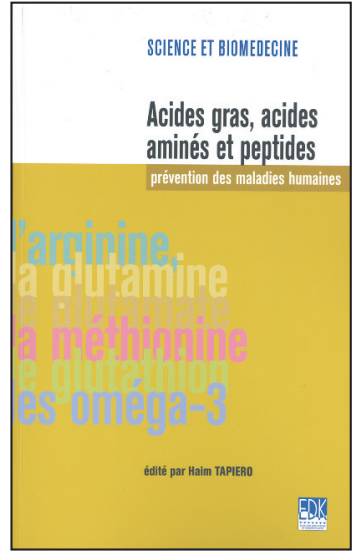

ISBN : 2-84254-108-1 80 pages

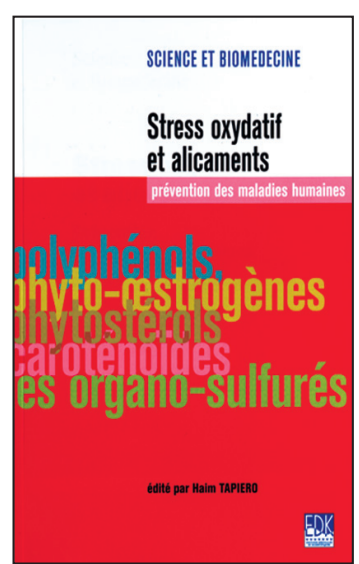

ISBN : 2-84254-111-1 86 pages

\section{Bon de commande}

À retourner à EDK, 25, rue Daviel - 75013 Paris

Tél. : 0158101905 - Fax : 0143293262 - E-mail : edk@edk.fr

NOM : Prénom :

Adresse :

Code postal :

Ville :

Pays :

Fonction :

Je souhaite recevoir l'ouvrage Les oligo-éléments : $10 €+3 €$ de port $=\mathbf{1 3} €$ TTC

Je souhaite recevoir l'ouvrage Acides gras, acides aminés et peptides : $12 €+3 €$ de port $=\mathbf{1 5} €$ TTC

Je souhaite recevoir l'ouvrage Stress oxydatif et alicaments : $14 €+3 €$ de port $=\mathbf{1 7} €$ TTC

en exemplaire, soit un total de $€$

$\square$ Par chèque, à l'ordre de $\mathbf{E} \mathbf{D} \mathbf{K}$

7 Par carte bancaire

\section{$\square$ Visa $\square$ Eurocard/Mastercard}

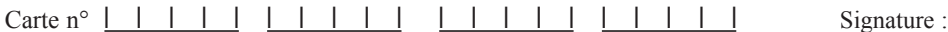

Date d'expiration :

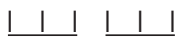

$N^{\circ}$ de contrôle au dos de la carte : $\quad$ | $\mid$ 